\title{
Molecular Docking and Molecular Dynamics Simulation of the Interaction of Cationic Imidazolium Porphyrin-Anthraquinone and Hsp90
}

\author{
Muhammad Arba ${ }^{1,2^{*}}$, Rahmana E. Kartasasmita ${ }^{1}$, Daryono H. Tjahjono ${ }^{1}$ \\ ${ }^{I}$ School of Pharmacy, Bandung Institute of Technology, Jalan Ganesha 10, Bandung 40132, Indonesia. \\ ${ }^{2}$ Department of Chemistry, Halu Oleo University, Jl. HEA Mokodompit Kendari, 93232, Indonesia.
}

Received: 10 September 2014 / Accepted: 30 November 2014

\begin{abstract}
:
Hsp90 is involved in the progressiveness of cancer cell through the activation of oncogenic client proteins, including Her2/ErbB2, Akt, Raf-1, and hTERT. Thus, targeting Hsp90 is considered as one of promising strategy in anti-cancer drug development. In the search of new potential Hsp90 inhibitors, three cationic imidazolium porphyrin-anthraquinone have been designed and their binding mode and affinity to Hsp90 were calculated employing AutoDock 4.2 and MM-PBSA method. All three ligands well fit into the binding site of Hsp90 and were able to interact with Hsp90 through hydrogen bond and additional $\pi$-cationic interactions, in which the latter was absent in the interaction of geldanamycin (GD). Molecular dynamics simulation confirmed the stability of each complex, and prediction of binding affinity using MMPBSA method show that porphyrin hybrids have comparable binding energies with that of GD.
\end{abstract}

Key words: Cationic porphyrin, Hsp90, MM-PBSA, in silico, molecular docking, molecular dynamics simulation

\section{Introduction}

Hsp90 is a molecular chaperone which is crucial in maintaining correct folding, maturation, and stabilization of client protein including those are involved in oncology [1]. Several oncogenic client proteins such as p53, Akt, Raf1, HER2 and EGFR are known to be highly dependent on Hsp90, indicating its crucial role in preserving cancer cell proliferation. Inhibition of Hsp90 function has been proven to have severe damage in multiple proteins involved in the hallmark of cancer [2$5]$.

Several Hsp90 inhibitors have been developed and enter preclinical trials including geldanamycin [6], which was then failed due to hepatotoxicity issue. Its successor, 17allylamino-17-demethoxygeldanamicyn (17AAG) entered Phase I trial [7], and then withdrawn because of solubility problem. Another analog of geldanamycin, 17DMAG [8], has also been developed and it shows to be more potent and more soluble than the previous one. The present study was devoted to designing new Hsp90 inhibitor based on porphyrin moiety by using in silico method combining molecular docking and molecular dynamics simulation. While molecular docking was utilized to predict binding mode of complex, molecular dynamics was used to monitor system stabilization during a period of time. Additionally, binding free energy of ligand to Hsp90 was calculated using MMPBSA method.

\section{Computational Methods}

Macromolecule and ligand preparations Crystal structure of the Hsp90 was taken from the Protein Data Bank (PDB ID of 1YET) [9]. Three porphyrin hybrids were designed, i.e. mono- $\mathrm{H}_{2} \mathrm{ImP}-\mathrm{AQ}$, bis- $\mathrm{H}_{2} \mathrm{ImP}-\mathrm{AQ}$, and tris- $\mathrm{H}_{2} \mathrm{ImP}-\mathrm{AQ}$ (Figure 1), which was built with the help of GaussView. Geometry optimization was performed on $3-21 \mathrm{G}^{*}$ basis set of the HF theory using Gaussian 09 software. The most stable conformation of each ligand was selected for initial docking.

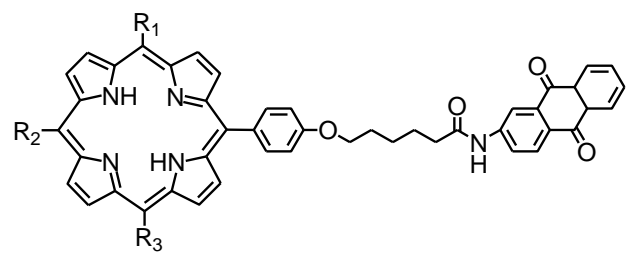

$$
\begin{aligned}
& \text { 1. } \mathrm{R}_{1}=\mathrm{R}_{3}=\mathrm{H}, \mathrm{R}_{2}=\text { Me-N } \hat{-}_{-}^{+} \cdot \mathrm{Me}: \text { mono- } \mathrm{H}_{2} \mathrm{ImP}-\mathrm{AQ} \\
& \text { 2. } \mathrm{R}_{2}=\mathrm{H}, \mathrm{R}_{1}=\mathrm{R}_{3}=\mathrm{Me}-\mathrm{N}_{\mathbf{-}}^{\hat{\mathrm{N}}^{+}} \cdot \mathrm{Me} \text { : bis- } \mathrm{H}_{2} \operatorname{ImP}-\mathrm{AQ}
\end{aligned}
$$

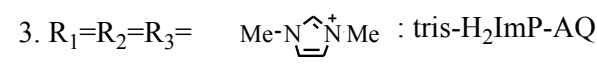

Figure 1. Structure of cationic imidazolium porphyrinanthraquinone. 


\section{Molecular Docking}

Exploration of binding modes of designed ligands to Hsp90 was achieved by using AutoDock $4.2[10,11]$. The binding pocket of Hsp90 was covered by the grid maps $62 \times 62 \times 62$ points of $0.375 \AA$ spacing. All docking parameters were used as the default values. Each docking simulation was performed at 100 runs.

\section{Molecular Dynamics Simulation}

Amber12 software was employed to study molecular dynamics simulations at three complexes of porphyrin hybrids plus the native ligand (GD) with Hsp90 obtained from docking structure. The Gaff and ff99SB force fields $[13,14]$ were used to parameterize the ligands and receptor, respectively. The partial atomic charges for ligands were assigned using AM1-BCC in Antechamber. The TIP3P water model was used to solvated the system with the distance of $10 \AA$ from the periphery of complex. The $\mathrm{Na}^{+}$ions was added to neutralize each system. The first energy minimization was carried out to minimize the water molecules by restraining the receptor and ligand with force constant of $500 \mathrm{kcal} / \mathrm{mol} \AA^{2}$. The second energy minimization was carried out to minimize water and side chains of the receptor by restraining backbone atoms of receptor with a force constant of 500 $\mathrm{kcal} / \mathrm{mol} \AA^{2}$. The final minimization was performed to minimize the entire system without any restraint. In each minimization, 500 steps steepest descents and 3500 steps of conjugate gradients were employed. Each system was then heated from 0 to $300 \mathrm{~K}$ for 100 ps with a time step of $0.0005 \mathrm{ps}$ and positional restraints for the backbone atoms of protein of $5 \mathrm{kcal} / \mathrm{mol} \AA 2$ in NVT ensemble. Then, equilibration of system was achieved at $300 \mathrm{~K}$ during two subsequent $50 \mathrm{ps}$ with each 3 and $1 \mathrm{kcal} / \mathrm{mol}$ $\AA 2$ force constants, which was then followed by 100 ps final equilibration without restraint. The MD simulation of $6 \mathrm{~ns}$ was performed using periodic boundary condition and constant number of particles, constant temperature of $300 \mathrm{~K}$ and constant pressure of $1 \mathrm{~atm}$ (NPT) ensemble. The Langevin coupling algorithm was used to deal with temperature regulation with a collision frequency of 1.0. The SHAKE algorithm was employed to constrain bond lengths involving hydrogen atoms [15] with 2 fs integration time step. PME method [16] was used to treat electrostatic interactions. The coordinate files were saved each 1 ps.

\section{MM/PBSA Calculations}

The binding free energy was calculated using MD trajectory of each system following the MM/PBSA method [18]. According to the method, the binding free energy is described as follows :

$$
\begin{aligned}
\Delta G_{\mathrm{bind}}= & G_{\text {complex }}-G_{\mathrm{rec}}-G_{\text {ligand }} \\
= & \Delta E_{\mathrm{MM}}+\Delta G_{\mathrm{PB}}+\Delta G_{\mathrm{SA}}-T \Delta S \\
\text { where } & \\
\Delta E_{\mathrm{MM}}= & \Delta E_{\text {bond }}-\Delta E_{\text {angle }}+\Delta E_{\text {torsion }}+\Delta E_{\mathrm{vdw}}+ \\
& \Delta E_{\mathrm{EEL}}
\end{aligned}
$$

and Gcomplex, Grec, and Gligand are free energy of complex, receptor, and ligand, respectively. $\triangle \mathrm{EMM}$ is the gas-phase interaction energy between the receptor and the ligand donated by van der Waals and electrostatic energies. $\triangle \mathrm{GPB}$ are the polar contributions to desolvation upon ligand-receptor binding interaction which is calculated by solving the Poisson_Boltzmann (PB) equation with a grid size of $0.5 \AA . \overline{\Delta G S A}$ is the nonpolar contributions to desolvation calculated by solvent accessible surface area (SASA) with solventprobe radius of $1.4 \AA$. $\mathrm{T} \Delta \mathrm{S}$ is the conformational entropy at temperature $\mathrm{T}$. Free energy calculations was performed based on 200 snapshots taken from 6-ns MD simulation trajectories.

\section{Results and Discussion}

The present study aimed to gain binding mode and affinity of porphyrin hybrids against Hsp90. Hsp90 is composed of N-terminal domain that contains an ATPbinding pocket, a middle domain, and $\mathrm{C}$-terminal region [19]. The residues of Hsp90 numbered 11 to 223, which was changed to be 1 to 213 in dynamics simulation. The ATP-binding pocket of N-terminal domain has an entrance of $12 \AA$ width and consists of polar Lys 58 and Lys 112 residues.

Porphyrin moiety along with side ring of imidazole is particularly interesting for its less than $12 \AA$ width and $\pi$ electron delocalization which are suitable for interaction with Lys58 and Lys 112 located in the entrance of Hsp90 binding cavity. While anthraquinone and the linker which both have oxygen and nitrogen atoms are suited to interact with the hydrophobic residues located in the bottom of the cavity such as Leu107, Gly135, Val136, Gly137.

The reliability of docking method was validated by redocking of native ligand (GD) of Hsp90. Figure 2 shows superimposition of GD in the crystal structure and after docking, which has RMSD of $0.935 \AA$.

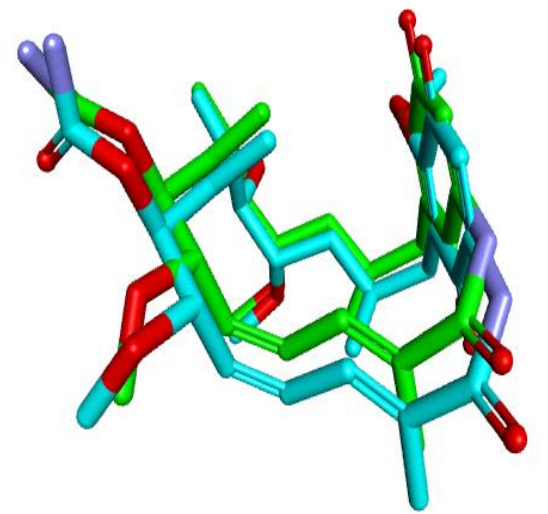

Figure 2. Superimposition of geldanamycin in the crystal structure (green) and after docking simulation (blue), with RMSD of $0.935 \AA$. 
Each of three compounds and natural ligand, GD, was docked into active site of Hsp90. The conformation with most negative of binding free energy was selected from the most populated cluster. Detailed molecular docking reveal that all ligands were well fit into the binding site of Hsp90. As in case of GD, each of tris-H2ImP-AQ and mono-H2ImP-AQ were able to establish hydrogen bond with Phe138 which was located in the bottom of binding cavity. Interestingly, additional pi-cationic interactions, which was absent in GD, were observed in the entrance of binding pocket between porphyrin macrocycle of trisH2ImP-AQ, bis-H2ImP-AQ and mono-H2ImP-AQ, with Lys58 and Lys112, respectively. The $\pi$-electron of anthraquinone was also detected to interact with Lys58 in tris-H2ImP-AQ. Figure 3 show 2D poses of each GD, tris-H2ImP-AQ, bis-H2ImP-AQ, and mono-H2ImP-AQ in Hsp90 binding site.
To further inspect stability of each binding modes between ligand and Hsp90 obtained from molecular docking, MD simulations of 6 ns was performed. Monitoring dynamic stability of each complex showed that all complexes seem to converge after 2 ns of the simulation and maintain their position during $6 \mathrm{~ns}$. Figure 4a display RMSD of Hsp90 backbone atoms relative to their starting structures of each complex, indicating their conformations have reached the equilibrium.

For detail observation of the residue fluctuation around their average position due to ligand binding of each complex during dynamics run, the Root-Mean-Square Fluctuation (RMSF) was calculated and presented in Figure 4b. a

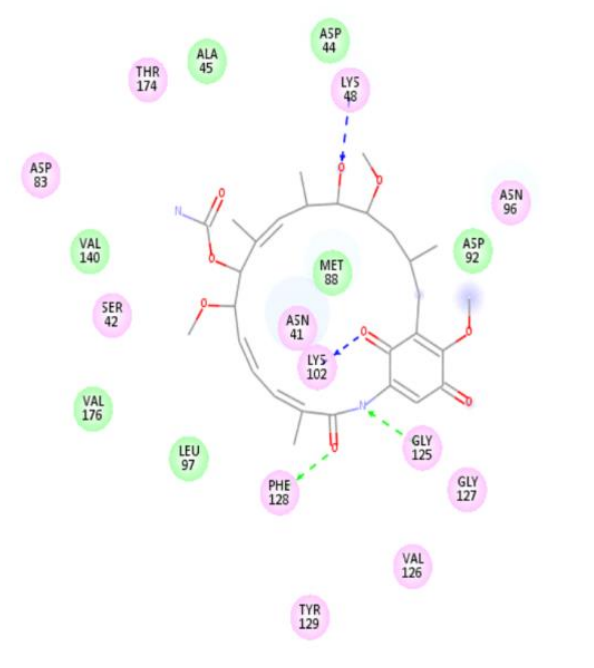

$\mathrm{c}$

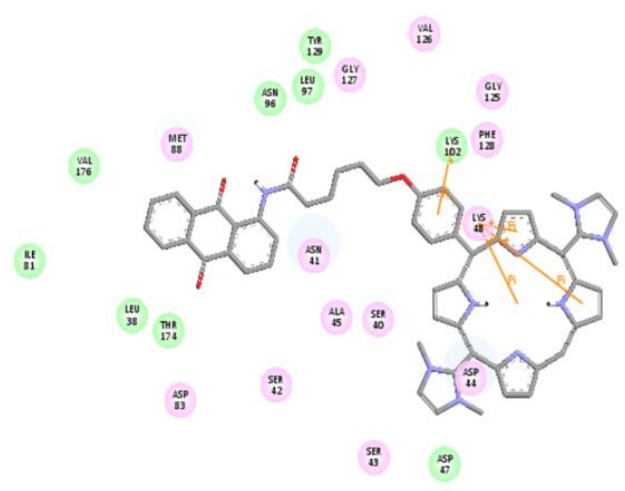

b

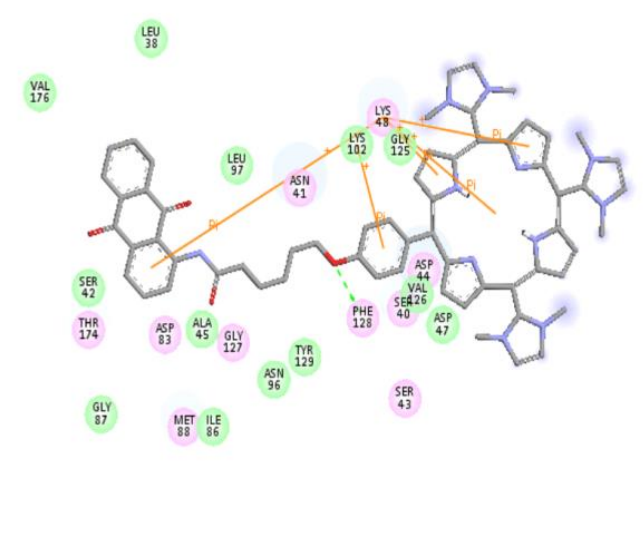

d

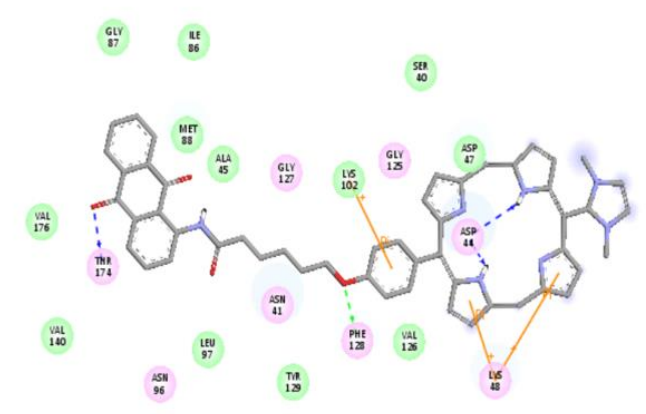

Figure 3. $2 \mathrm{D}$ poses of GD (a), tris- $\mathrm{H}_{2} \operatorname{ImP}-\mathrm{AQ}$ (b), bis- $\mathrm{H}_{2} \operatorname{ImP}-\mathrm{AQ}$ (c), and mono- $\mathrm{H}_{2} \mathrm{ImP}-\mathrm{AQ}$ (d) in Hsp90 binding site. 


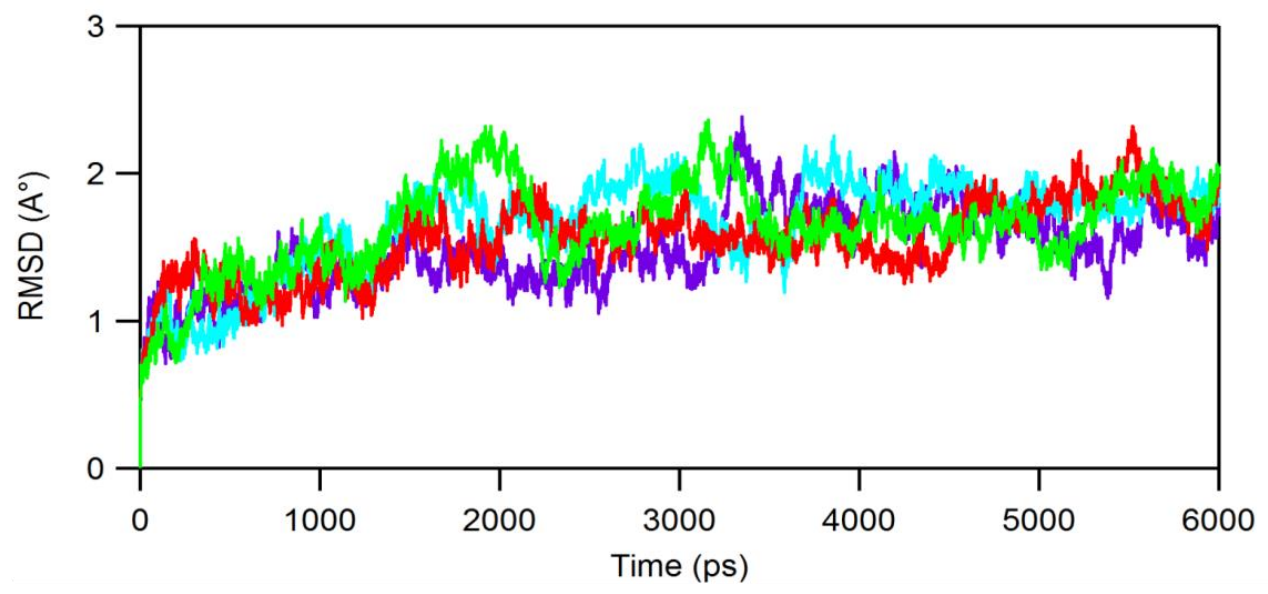

b

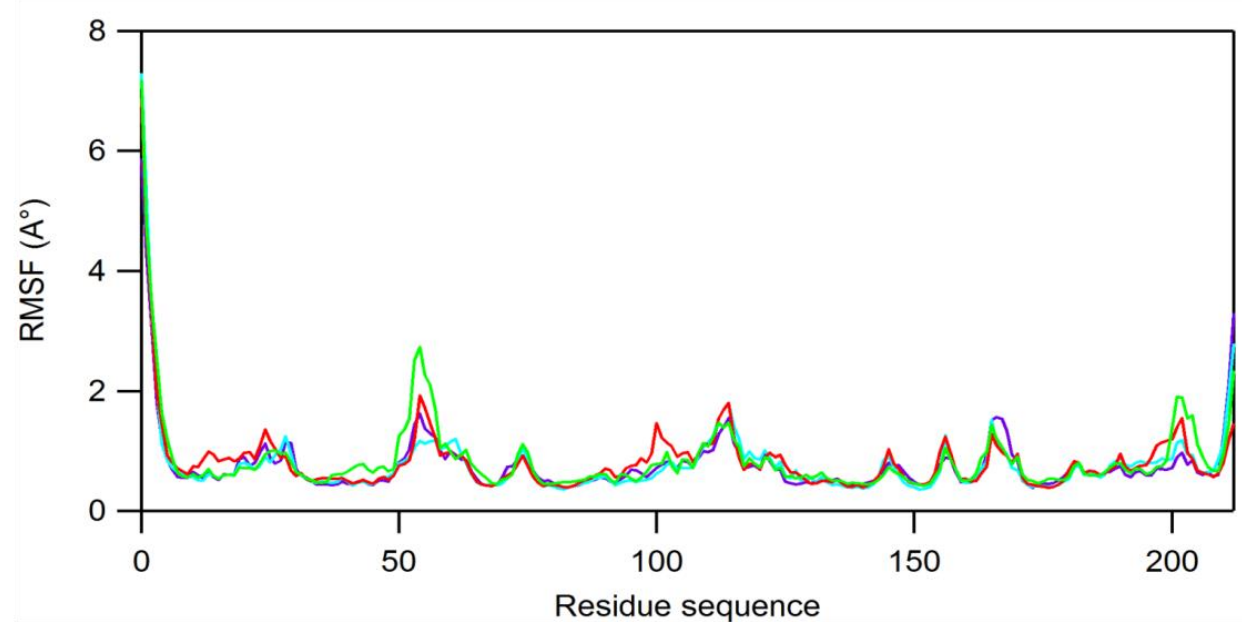

Figure 4. RMSD of Hsp90 backbone atoms relative to their starting structures of each complex (a), and the root-mean-square fluctuation (RMSF) (b), in which tris- $\mathrm{H}_{2} \mathrm{ImP}-\mathrm{AQ}$ assigned as blue, bis- $\mathrm{H}_{2} \mathrm{ImP}-\mathrm{AQ}$ as red, mono- $\mathrm{H}_{2} \mathrm{ImP}-\mathrm{AQ}$ as green, and GD as purple colors.

Table 1. The binding free energy components of each ligand to Hsp90

\begin{tabular}{lccccccc}
\hline Ligand & $\boldsymbol{\Delta} \boldsymbol{E}_{\text {VDW }}$ & $\boldsymbol{\Delta} \boldsymbol{E}_{\text {ELE }}$ & $\boldsymbol{\Delta} \boldsymbol{E}_{\text {INT }}$ & $\boldsymbol{\Delta} \boldsymbol{E}_{\mathrm{PBCAL}}$ & $\boldsymbol{\Delta} \boldsymbol{E}_{\text {PBSUR }}$ & $\boldsymbol{\Delta} \boldsymbol{E}_{\mathrm{PBELE}}$ & $\boldsymbol{\Delta} \boldsymbol{E}_{\mathrm{PBTOT}}$ \\
\hline mono-H ImP-AQ & -62.92 & -167.18 & -0.00 & 208.00 & -8.86 & 40.83 & -30.95 \\
bis-H ImP-AQ $_{2}$ & -66.40 & -281.49 & -0.00 & 324.66 & -9.12 & 43.17 & -32.35 \\
tris-H 2 ImP-AQ & -69.66 & -424.92 & -0.00 & 470.09 & -9.73 & 45.16 & -34.23 \\
GD & -52.20 & -43.340 & -0.00 & 70.680 & -7.59 & 27.33 & -32.46 \\
\hline
\end{tabular}

All complexes showed similar RMSF distributions, indicating that all porphyrin hybrids have similar interaction modes as the natural ligand on whole. A remarkably high fluctuation was noted for residues at the $\mathrm{N}$ - and C-terminals and loop regions. A stable RMSF was observed in residue Phe138, Asp54, Lys58, and Lys 112, in which ligand establish hydrogen bond and or pi-cationic interactions.

\section{Free Energy Calculations}

Table 1 shows the binding free energy components of hybrid porphyrin to Hsp90. The electrostatic energies $(\triangle E E L E)$ of porphyrin hybrids are much lower than that of GD, which are markedly favoured for all complexes.

The favorable contribution is also appear from van der Waals energy $(\triangle E V D W)$, in which porphyrin hybrids has slightly lower energies than that of GD. The slightly favorable contribution arose from the nonpolar contribution to solvation energy ( $\triangle E P B S U R)$, in which 
the four complexes were not greatly different. The electrostatic term to solvation energy ( $\triangle \mathrm{EPBCAL})$ of porphyrin hybrids was much more positive than that of GD, which was all greatly unfavorable. The summation of $\triangle E E L E$ and $\triangle E P B C A L$ contributions, $\triangle E P B E L E$, is higher unfavorable in porphyrin hybrids than that of GD, to the total binding free energy for all complexes. In addition, stabilization of each complex by ligands seems to be not greatly affected by number of side ring substituent of imidazole.

\section{Conclusions}

The new scaffold, porphyrin-anthraquinone, is evident by using in silico method, to have potential as new Hsp90 inhibitor. The binding free energies of the ligands in the active site of Hsp90 are comparable with the known inhibitor, GD, reserving their potential to be continued in further study.

\section{References}

[1] D.D. Mosser, R.I. Morimoto, Molecular chaperones and the stress of oncogenesis, Oncogene, 23, 2004, 2907-2918.

[2] L. Whitesell, S.L. Lindquist, HSP90 and the chaperoning of cancer, Nat. Rev. Cancer, 5, 2005, 761-772.

[3] A. Maloney, and P. Workman, HSP90 as a new therapeutic target for cancer therapy: the story unfolds, Expert Opin. Biol. Ther., 2, 2002, 3-24.

[4] A. Kamal, M.F. Boehm, F.J. Burrows, Therapeutic and diagnostic implications of Hsp90 activation, Trends Mol. Med., 10, 2004, 283-290.

[5] G. Chiosis, M. Vilenchik, J. Kim, and D. Solit, Hsp90: the vulnerable chaperone, Drug Discov. Today, 9, 2004, 881888 .

[6] L. Neckers, T.W. Schulte, and E. Mimnaugh, Geldanamycin as a potential anticancer agent: its molecular target and biochemical activity, Invest. New Drug, 17, 1999, 361-373.

[7] T.W. Schulte, and L.M. Neckers, The benzoquinone ansamycin 17-allylamino-17-demethoxygeldanamycin binds to HSP90 and shares important biologic activities with geldanamycin, Cancer Chemother. Pharmacol., 42, 1998, 273-279.

[8] G. Kaur, D. Belotti, A.M. Burger, K. Fisher-Nielson, P. Borsotti, E. Riccardi, J. Thillainathan, M. Hollingshead, E.A.
Sausville, and R. Giavazzi, Antiangiogenic properties of $17-$ (dimethylaminoethylamino)-17-demethoxygeldanamycin: an orally bioavailable heat shock protein 90 modulator, Clin. Cancer Res., 10, 2004, 4813-4821.

[9] C.E. Stebbins, A.A. Russo, C. Schneider, N. Rosen, F.U. Hartl, and N.P Pavletich, Crystal structure of an Hsp90 geldanamycin complex: targeting of a protein chaperone by an antitumor agent, Cell, 89, 1997, 239-250.

[10] D.S. Goodsell, G.M. Morris, and A.J. Olson, Automated docking of flexible ligands: applications of autodock, J. Mol. Recog., 9, 1996, 1-5.

[11] G.M. Morris, D.S. Goodsell, R.S. Halliday, R. Huey, W.E. Hart, R.K. Belew, and A.J. Olson, Automated docking using a Lamarckian genetic algorithm and an empirical binding free energy function, J. Comput. Chem., 19, 1998, 1639-1662.

[12] D.A. Case, T.E. Cheatham, T. Darden, H. Gohlke, R. Luo, K.M. Merz, A. Onufriev, C. Simmerling, B. Wang, and R.J. Woods, The Amber biomolecular simulation programs, $J$. Comput. Chem., 26(16), 2005, 1668-1688.

[13] H. Viktor, A. Robert, O. Asim, S. Bentley, R. Adrian, and C. Simmerling, Comparison of multiple Amber force fields and development of improved protein backbone parameters, Proteins, 65(3), 2006, 712-725.

[14] J. Wang, R.M. Wolf, J.W. Caldwell, P.A. Kollman, and D.A. Case, Development and testing of a general amber force field, J. Comput. Chem., 25, 2004, 1157-1174.

[15] J-P. Ryckaert, G. Ciccotti, and H.J.C. Berendsen, Numerical integration of the cartesian equations of motion of a system with constraints: molecular dynamics of n-alkanes, $J$. Comput. Phys., 23, 1977, 327-341.

[16] T. Darden, D. York, and L. Pedersen, Particle mesh Ewald: An $\mathrm{N} \cdot \log (\mathrm{N})$ method for Ewald sums in large systems, $J$. Chem. Phys., 98, 1993, 10089-10092.

[17] W. Humphrey, A. Dalke, and K. Schulten, VMD-Visual molecular dynamics, J. Mol. Graphics., 14, 1996, 33-38.

[18] P.A. Kollman, I. Massova, C. Reyes, B. Kuhn, S. Huo, L. Chong, M. Lee, T. Lee, Y. Duan, W. Wang, O. Donini, P. Cieplak, J. Srinivasan, D.A. Case, and T.E. Cheatham, Calculating structures and free energies of complex molecules: combining molecular mechanics and continuum models, Accounts Chem. Res., 33, 2000, 889-897.

[19] J. Liu, F. Wang, Z. Ma, X. Wang, and Y. Wang, Structural determination of three different series of compounds as Hsp90 inhibitors using 3D-QSAR modeling, molecular docking and molecular dynamics methods, Int. J. Mol. Sci., 12, 2011, 946-970. 